\title{
Nanoparticle SN-38-encapsulated Micelle Formulation IT-141
}

National Cancer Institute

\section{Source}

National Cancer Institute. Nanoparticle SN-38-encapsulated Micelle Formulation IT-141. NCl Thesaurus. Code C148511.

A nanoparticle-based formulation consisting of polymeric micelles, composed of triblock copolymers, loaded with the bioactive hydrophobic irinotecan metabolite 7-ethyl-10hydroxy-camptothecin (SN-38), with potential antineoplastic activity. Upon administration, nanoparticle SN-38-encapsulated micelle formulation IT 141 diffuses into the tumor site and the $\mathrm{SN}-38$ moiety binds to and inhibits topoisomerase I by stabilizing the cleavable complex between topoisomerase I and DNA, which results in DNA breaks, inhibition of DNA replication, and apoptosis. Compared to SN-38 alone, this formulation increases the water solubility of SN-38 and allows the delivery of higher doses of SN-38. IT-141 also has a much longer circulation time and reduces toxicity to normal, healthy tissues. 